\title{
Asociación entre variables sociodemográficas, clínicas y calidad de vida con el apoyo social percibido en pacientes colombianos con cáncer
}

\author{
Association Between Sociodemographic, Clinical Uariables and Quality of Life \\ with Social Support Perceived in Colombian Patients with Cancer \\ Associação entre variáueis sociodemográficas, clínicas e qualidade de vida \\ com 0 apoio social percebido em pacientes colombianos com cancro
}

\author{
Luis Fernando Fontibón*, Lorena Acosta Hernández*, Juliet Valdelamar Jiménez*, \\ Adriana Valdelamar Jiménez* y Ricardo Sánchez Pedraza* \\ Instituto Nacional de Cancerología ESE, Colombia
}

Doi: dx.doi.org/10.12804/apl33.03.2015.02

\section{Resumen}

Introducción: Estudios previos han encontrado un nexo entre calidad de vida relacionada con la salud y el apoyo social percibido (ASP). Métodos: Estudio transversal-analítico para identificar la asociación entre variables sociodemográficas, clínicas y la calidad de vida con el ASP en pacientes con diferentes tipos de cáncer. Se evaluaron 119 pacientes mediante el cuestionario Medical Outcomes Study-Social Support Survey (MOS-SSS) y la Functional Assessment of Cancer Therapy-General (FACT-G) mediante entrevista directa o telefónicamente. Para el análisis se manejaron herramientas para evaluar patrones de correlación y se utilizaron métodos de regresión lineal. Resultados: No se encontraron diferencias significativas entre el MOS y las variables sexo, estrato socioeconómico y estado civil. En relación con el modelo de regresión se encontraron coeficientes signi- ficativos en las variables bienestar funcional y sociofamiliar (dimensiones de la FACT-G) y cáncer de mama. La presencia de comorbilidad se relaciona con mayor puntaje de la escala MOS, lo mismo que lo hacen las dimensiones de la escala FACT-G. Conclusión: El ASP es una variable que interactúa de manera positiva con el constructo de calidad de vida en pacientes oncológicos. Palabras clave: apoyo social; calidad de vida; cáncer; MOS; FACT-G.

\section{fbstract}

Introduction: Previous studies have found a relationship between quality of life related to health and perceived social support (PSS). Methods: Cross-sectional, analytical study identifying association between sociodemographic, clinical and quality of life variables with the PSS in patients with different types of cancer. One hundred

* Luis Fernando Fontibón, Lorena Acosta Hernández, Juliet Valdelamar Jiménez, Adriana Valdelamar Jiménez, Investigaciones Clínicas, Instituto Nacional de Cancerología ESE; Ricardo Sánchez Pedraza, Facultad de Medicina, Universidad Nacional de Colombia e Investigaciones Clínicas, Instituto Nacional de Cancerología ESE.

La correspondencia relacionada con este artículo debe ser enviada a Luis Fernando Fontibón, Instituto Nacional de Cancerología, Investigaciones Clínicas. Correo electrónico: 1ffontibon@cancer.gov.co

Cómo citar este artículo: Fontibón L. F., Acosta, L., Valdelamar, J., Valdelamar, A. \& Sánchez, R. (2015). Asociación entre variables sociodemográficas, clínicas y calidad de vida con el apoyo social percibido en pacientes colombianos con cáncer. Avances en Psicología Latinoamericana, 33(3), 387-395. doi: dx.doi.org/10.12804/apl33.03.2015.02 
and nineteen patients were evaluated by the questionnaire Medical Outcomes Study Social Support Survey (MOS-SSS) and the Functional Assessment of Cancer Therapy - General (FACT-G) by direct or telephone interview. Data analysis included correlation pattern identification and linear regression. Results: No significant differences between the MOS and categorical variables sex, socioeconomic and marital status were found. Regarding the regression analysis, significant coefficients were found for the variables functional well-being and social well-being (dimensions of the FACT-G) and breast cancer. Comorbidity is associated with higher scores on the MOS scale, as are the dimensions of the FACT-G scale. Conclusion: The PSS interacts positively with the construct of quality of life in cancer patients. Keywords: social support; quality of life; cancer; MOS; FACT-G.

\section{Resumo}

Introdução: estudos prévios têm encontrado nexorelação entre qualidade de vida relacionada com a saúde e o apoio social percebido (ASP). Métodos: estudo transversal- analítico para identificar associação entre variáveis sociodemográficas, clínicas e a qualidade de vida com o ASP em pacientes com diferentes tipos de cancro. Avaliaram- se 119 pacientes mediante o questionário Medical Outcomes Study-Social Support Survey (MOS-SSS) e a Functional Assessment of Cancer Therapy - General (FACT-G) mediante entrevista direta ou telefonicamente. Para a análise empregaram-se ferramentas para avaliar patrões de correlação e utilizaram-se métodos de regressão lineal. Resultados: não se encontraram diferenças significativas entre o MOS e as variáveis gênero, estrato socioeconómico e estado civil. Em relação ao modelo de regressão encontraram-se coeficientes significativos nas variáveis bem-estar funcional e sociofamiliar (dimensões da FACIT-G) e cancro de mama. A presençcia de comorbidade relaciona-se com maior pontuação da escala MOS, igualmente presente nas dimensões da escala FACIT-G. Conclusões: OASPé uma variável que interatua de forma positiva com o construto de qualidade de vida em pacientes oncológicos.
Palavras-chave: apoio social, qualidade de vida, cancro, MOS, FACT-G.

En la investigación sobre cáncer se ha destacado la importancia de los aspectos psicosociales (Arias et al., 2008), en especial lo concerniente a la calidad de vida relacionada con la salud. La Organización Mundial de la Salud ha definido la calidad de vida como la percepción del individuo sobre su posición en la vida dentro del contexto cultural y el sistema de valores en el que vive y con respecto a sus metas, expectativas, normas y preocupaciones. Es un concepto extenso y complejo que engloba la salud física, el estado psicológico, el grado de independencia, las relaciones sociales, las creencias personales y la relación con las características sobresalientes del entorno (World Health Organization, 1997).

Entre los factores que predicen el puntaje de la calidad de vida en pacientes con cáncer se encuentran variables biológicas, psicológicas y sociales, como el tamaño del tumor, el bienestar espiritual, los ingresos, los trastornos del estado de ánimo y el apoyo social (Dapueto, Servente, Francolino \& Hahn, 2005). La presencia de malestar psicológico, el apoyo social, así como la funcionalidad de los pacientes, se asocia tanto al diagnóstico de la enfermedad como a los tratamientos empleados (Nausheen, Gidron, Peveler \& Moss-Morris, 2009).

En pacientes con enfermedades crónicas como el cáncer, el apoyo social se entiende como una necesidad que permite mitigar el efecto de los cambios asociados a la enfermedad; por esta razón se relaciona con la calidad de vida de dos formas: (a) reduciendo el impacto psicológico de los acontecimientos estresantes en la vida de la persona y el estrés que pueda contribuir a la aparición o agravamiento de la enfermedad y (b) influyendo directamente en la enfermedad física, lo que contribuye a una menor morbilidad y, sobre todo, a una mejor rehabilitación (Rodríguez, Pastor \& López, 1993). El estudio del apoyo social 
difiere en la investigación según los instrumentos utilizados para su medición, su conceptualización y operacionalización, por lo cual ha de abordarse como un constructo multidimensional que abarca aspectos cuantitativos (estructurales) que alude a los recursos que lo posibilitan; usualmente medido por el número de personas o tamaño de la red social y aspectos cualitativos (funcionales) que comprende la percepción que se tiene de las relaciones interpersonales y la utilidad que representan (Costa, Salamero \& Gil, 2007).

La relación entre apoyo social y salud ha sido ampliamente estudiada, y se han consolidado los hallazgos sobre los beneficios para la salud y el bienestar que comportan (Evon, Esserman, Ramcharran, Bonner \& Fried, 2011; Fagundes et al., 2012; Terol, 1999; Waters, Liu, Schootman \& Jeffe, 2013). Los adultos con un historial de apoyo social han reportado tener relaciones más cercanas y duraderas, se muestran como miembros de una red más fiable y sensible en tiempos de necesidad (Gottlieb \& Bergen, 2010) o en momentos críticos, como antes y después de un procedimiento quirúrgico (Nausheen et al., 2009) o ante el diagnóstico de una enfermedad como el cáncer (Thompson, Rodebaugh, Pérez, Schootman \& Jeffe, 2013). Por el contrario, personas socialmente aisladas son comúnmente relacionadas con prácticas poco saludables, como tomar, fumar, presentar "atracones de comida" o ausencia de ejercicio físico, que se relacionan con incrementos en la vulnerabilidad a través del cáncer y su asociación a mortalidad (Nausheen et al., 2009).

En estudios previos se han identificado variables sociodemográficas y psicosociales que se asocian o predicen el apoyo social percibido, por ejemplo, la edad, el sexo, el nivel socioeconómico, la raza, el lugar de residencia (urbana $v s$. rural), la depresión y la ansiedad (Fagundes et al., 2012; Heinonen et al., 2001; Mickelson \& Kubzansky, 2003; Thompson et al., 2013; Waters et al., 2013), las cuales deben considerarse factores intervinientes en el manejo del paciente oncológico y crónico. En Colombia no existen estudios sistemáticos al respecto y es poca la literatura sobre el papel del apoyo social en la calidad de vida en pacientes con cáncer; por ello es indispensable conocer el tipo de relaciones que se presentan entre dichas variables. Este estudio, en consecuencia, busca identificar la asociación que se pueda dar entre variables sociodemográficas y clínicas y la calidad de vida con el apoyo social percibido en una muestra de pacientes colombianos con diferentes tipos de cáncer.

\section{Materiales y métodos}

Se trata de un estudio transversal-analítico realizado en el Instituto Nacional de Cancerología ESE de la ciudad de Bogotá, el cual recibió la aprobación del Comité de Ética del Instituto.

\section{Participantes}

Se evaluaron 119 pacientes, de nacionalidad colombiana, mayores de 18 años de edad, con diagnóstico confirmado histopatológicamente de cáncer de mama, cuello uterino, próstata, estómago y colon-recto, candidatos a cirugía como principal alternativa de manejo y con intención curativa. Se excluyeron aquellos pacientes que presentaban alteraciones sensoriales o cognitivas que les impedían responder adecuadamente las escalas y pacientes que presentaban otras neoplasias concomitantes o quienes habían sido intervenidos quirúrgicamente previamente por la patología oncológica. La búsqueda de pacientes se realizó mediante la historia clínica; luego se contactaron personalmente en las consultas del Instituto o vía telefónica por un entrevistador, quien después de obtener el consentimiento informado aplicó las escalas correspondientes.

\section{Instrumentos}

Durante la entrevista se recogieron los datos sociodemográficos referidos a sexo, edad, estrato 
socioeconómico, escolaridad (medida en años) y estado civil. Se aplicó el cuestionario Medical Outcomes Study-Social Support Survey (MOS-SSS) (Sherbourne \& Stewart, 1991), desarrollado para evaluar el apoyo social percibido por pacientes con enfermedades crónicas. La versión original consta de 20 ítems, donde el primero registra la cantidad de personas que conforman la red social y los 19 restantes evalúan diferentes aspectos del apoyo social: apoyo emocional/informacional (ítems 3, $4,8,9,13,16,17$ y 19), apoyo instrumental (ítems $2,5,12$ y 15), interacción social positiva (ítems 7 , 11,14 y 18) y apoyo afectivo (ítems 6,10 y 20). El cuestionario tiene como opciones de respuesta una escala Likert de 5 puntos, siendo 1 "nunca" y 5 "siempre", y considerando que a mayor puntaje hay un mayor apoyo social percibido.

El MOS ha pasado por varios estudios de validación y adaptación transcultural (Alonso, Menéndez \& Graça, 2007; Rodríguez, Rodríguez \& Camelo, 2007); pero ha sido de interés particular la validación realizada en Colombia por Londoño et al. (2012) en personas sanas, que arrojó una adecuada consistencia interna (alfa de Cronbach de .941) para el total de la escala, además el análisis factorial confirmatorio mostró buenos índices de bondad de ajuste para tres factores. El MOS también se ha usado en poblaciones con cáncer y otras patologías (Thompson et al., 2013).

Se aplicó la escala de calidad de vida relacionada a la salud, desarrollada por la organización Functional Assessment for Chronic Illness Therapy y validada en Colombia, que mostró una adecuada consistencia interna (alfa de Cronbach de .89). La confiabilidad test-retest arrojó coeficientes de correlación en un rango de .64-.76, y la validez de criterio obtenida con base en la comparación con la EORTC QLQC-30 arrojó valores de correlación que oscilaron entre .1 y .7 en las subescalas contrastadas (Sánchez, Ballesteros \& Arnold, 2011). La Functional Assessment of Cancer Therapy-General (FACT-G) es una medición de la calidad de vida relacionada con la salud en pacientes con cáncer. Este cuestionario contiene 4 subescalas con 27 ítems en total: estado físico general de la salud (GP), ambiente familiar y social (GS), estado emocional (GE) y capacidad de funcionamiento personal (GF).

\section{Análisis estadístico}

Para el análisis descriptivo de las variables continuas se utilizaron medias (con desviación estándar como medida de variabilidad) o medianas (con rangos como medida de variabilidad). Las variables categóricas se resumieron utilizando porcentajes.

Se compararon las medias del puntaje de la escala MOS entre modalidades de las variables categóricas consideradas en el estudio; para esto se utilizaron pruebas $t$ manejando hipótesis a dos colas.

Para evaluar las variables relacionadas con el apoyo social, medido con la escala MOS, se utilizó un modelo de regresión lineal en el cual se tomó el puntaje total de la escala MOS como variable dependiente y se incorporaron como variables independientes el sexo, la edad, el estrato socioeconómico - tomado en dos categorías: 1 y 2 (niveles socioeconómicos bajos) vs. 3 y 4 (niveles socioeconómicos medios) - el estado civil (tomado como presencia de compañero estable), la presencia de comorbilidad (sí o no) el tipo de cáncer (manejada como variable indicadora se tomó como categoría de referencia el cáncer de próstata) y los puntajes de los dominios de la escala FACT-G de calidad de vida (bienestar físico, socio familiar, emocional y funcional).

Para la selección del modelo que mejor explicara el puntaje en la escala MOS se utilizó un método stepwise con probabilidad de entrada al modelo de .2. Los supuestos del modelo se verificaron con herramientas gráficas basadas en residuos.

El análisis de los datos se realizó con el programa Stata $13 \AA$. Para las pruebas de hipótesis se utilizó un nivel de significación del 5\%. 


\section{Resultados}

Se evaluaron un total de 119 pacientes que tuvieron una media de edad de 59.6 años $(D E=16.3)$. El 58\% fueron mujeres $(N=69)$. Se encontró una mediana de escolaridad de 6 años cursados (intervalo entre 0 y 22 años). El estrato socioeconómico más frecuente fue el $2(42.86 \% ; N=51)$, seguido del $3(35.29 \% ; N=42)$, del $1(15.97 \% ; N=19)$ y del $4(5.88 \% ; N=7)$. En relación con el estado civil, un $66.39 \%$ de los pacientes tenía una relación de pareja estable $(N=79)$. Los pacientes tuvieron diagnóstico confirmado histopatológicamente en las siguientes localizaciones: mama $(35.29 \% ; N=$ 42), estómago $(21.85 \% ; N=26)$, próstata $(18.49 \%$; $N=22)$, colon-recto $(15.13 \% ; N=18)$ y cuello uterino $(9.24 \% ; N=11)$. Un $32,8 \%$ de los pacientes tuvo algún tipo de comorbilidad $(N=39)$. De los pacientes con comorbilidad, 13 tuvieron 2 patologías comórbidas concomitantemente y 5 tuvieron 3 patologías simultáneas.

Las comorbilidades más frecuentes fueron de tipo arterial periférico (tabla 1). El puntaje de la escala MOS tuvo una media de $77.6(D E=13,5)$. Esta escala puede tomar rangos entre 19 y 95 . Los valores de los puntajes de la escala MOS según las modalidades de las variables categóricas incluidas

Tabla 1

Tipo de comorbilidades presentes

en los pacientes de la muestra

\begin{tabular}{lcc}
\hline \multicolumn{1}{c}{ Comorbilidad 1 } & $N$ & \multicolumn{1}{c}{$\%$} \\
\hline Enfermedad arterial periférica & 17 & 27.87 \\
Insuficiencia cardiaca & 13 & 21.31 \\
Enfermedad respiratoria crónica & 10 & 16.39 \\
Úlcera gastroduodenal & 8 & 13.11 \\
Enfermedad del tejido conectivo & 6 & 9.84 \\
Diabetes & 5 & 8.20 \\
Infarto de miocardio & 1 & 1.64 \\
Enfermedad cerebro-vascular & 1 & 1.64 \\
Total eventos & 61 & 100.00 \\
\hline
\end{tabular}

en el estudio se presentan en la tabla 2 (no se encontraron diferencias significativas). La relación entre las variables continuas (edad, años de escolaridad y los dominios de la escala FACT-G) se muestran en la figura 1. Puede verse que los patrones de relación lineal se insinúan en las variables relacionadas con la escala FACT-G.

En relación con el modelo de regresión lineal, se encontraron coeficientes significativos en algunas de las variables (tabla 3): bienestar

Tabla 2

Puntaje de escala MOS según variables categóricas

\begin{tabular}{llcc}
\hline & & Media & $D E$ \\
\hline \multirow{2}{*}{ Sexo } & $\mathrm{F}$ & 76.42 & 13.43 \\
& $\mathrm{M}$ & 79.36 & 13.57 \\
Estrato socioeco- & Estratos 1 y 2 & 77.51 & 14.15 \\
nómico & Estratos 3 y 4 & 77.86 & 12.67 \\
& Sin pareja & 76.60 & 13.32 \\
Estado civil & Con pareja & 78.19 & 13.66 \\
\hline
\end{tabular}

Tabla 3

Modelo de regresión lineal para el puntaje de la escala MOS

\begin{tabular}{|c|c|c|c|}
\hline & Coeficiente & $p>t$ & IC $95 \%$ \\
\hline Sexo & -2.54 & .50 & $-9.99-4.91$ \\
\hline Edad & .02 & .73 & $-.12-.17$ \\
\hline Estrato socioeconómico & -.25 & .92 & $-5.00-4.50$ \\
\hline Estado civil & -2.14 & .41 & $-7.20-2.93$ \\
\hline Escolaridad & .23 & .39 & $-.31-.79$ \\
\hline Comorbilidad & 3.35 & .17 & $-1.48-8.18$ \\
\hline Cáncer de cuello uterino* & -3.01 & .61 & $-14.69-8.67$ \\
\hline Cáncer colorrectal ${ }^{*}$ & -6.21 & .15 & $-14.79-2.36$ \\
\hline Cáncer gástrico* & -3.27 & .43 & $-11.38-4.84$ \\
\hline Cáncer de mama* & -11.32 & .02 & $-2.94-1.70$ \\
\hline Bienestar físico & -.18 & .53 & $-.77-.40$ \\
\hline Bienestar sociofamiliar & 1.10 & .00 & $.55-1.64$ \\
\hline Bienestar emocional & .02 & .94 & $-.44-.48$ \\
\hline Bienestar funcional & .84 & .01 & $.21-1.48$ \\
\hline
\end{tabular}




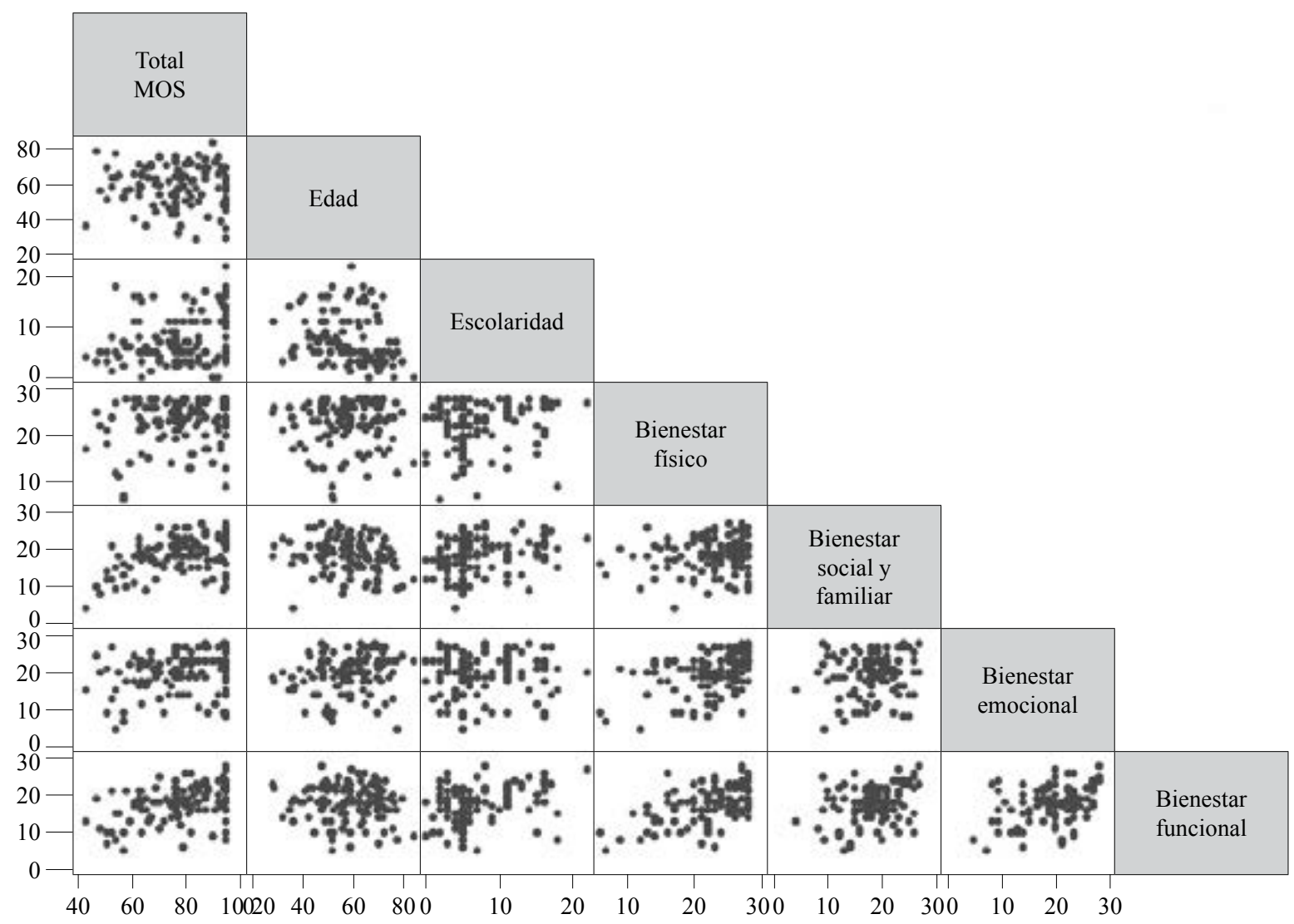

Figura 1. Relación entre variables continuas incluidas en el modelo de regresión

funcional, bienestar sociofamiliar (estas dos, dimensiones de la escala FACT-G de calidad de vida) y cáncer de mama. El método de selección del mejor modelo indica que, en comparación con tener cáncer de próstata, tener cáncer de mama disminuye en casi 6 puntos el puntaje total de la escala MOS, habiendo ajustado por las otras variables del modelo (bienestar sociofamiliar, bienestar funcional y presencia de comorbilidad). La presencia de comorbilidad aumenta el puntaje de la escala MOS en casi 4 puntos. Las dimensiones de la escala FACT-G que quedaron incluidas en este modelo de predicción (tabla 4) también intervienen aumentando el puntaje en la escala MOS (por cada aumento en los puntajes de estas dimensiones se aumenta casi un punto en la escala MOS).
Tabla 4

Modelo que mejor predice el puntaje en la escala MOS

\begin{tabular}{lrrr}
\hline & Coeficiente & $p>t$ & \multicolumn{1}{c}{ IC 95\% } \\
\hline Bienestar sociofamiliar & .98 & .00 & $.48-1.48$ \\
Bienestar funcional & .79 & .00 & $.30-1.28$ \\
Cáncer de mama* & -5.86 & .01 & $-1.38-1.35$ \\
Comorbilidad & 3.95 & .09 & $-.64-8.55$
\end{tabular}

*Cáncer de próstata fue tomado como categoría de referencia.

\section{Discusión}

El objetivo principal del presente estudio fue observar los factores que se relacionaban con el apoyo social percibido en pacientes con cáncer de mama, cuello uterino, próstata, estómago y colon-recto. Dentro de los principales hallazgos se 
observó que no existieron diferencias significativas en el MOS respecto a las variables categóricas sexo, estrato socioeconómico y estado civil, a pesar de que Heinonen et al. (2001) afirman que los hombres están menos satisfechos con su apoyo social sin importar su estado marital. A la vez, Thompson et al. (2013) observaron una asociación entre personas que están casadas o conviviendo con un compañero permanente con un apoyo social elevado. Mickelson y Kubzansky (2003) y Fagundes et al. (2012) encontraron que el nivel socioeconómico sí se relaciona con el apoyo social; personas con un menor nivel socioeconómico presentaban menor apoyo social percibido, además de menor contacto con amigos. Por otra parte, adultos con menores ingresos, baja educación y trabajos de menor prestigio presentan mayores riesgo de enfermedad crónica, discapacidad y mortalidad prematura (Fagundes et al., 2012; Mickelson \& Kubzansky, 2003).

Por otra parte, no se presenta relación en cuanto a la edad y la escolaridad; sin embargo, diversos estudios han encontrado, por lo menos, asociación entre dichas variables: a mayor edad y escolaridad, mejor apoyo social (Fagundes et al., 2012; Waters et al., 2013).

Los patrones de relación lineal solo se insinúan en las variables relacionadas con la FACT-G, hallazgos sustentados por diversos estudios (Cheng et al., 2013; Costa, Ballesteros \& Gil, 2014; Huang \& Hsu, 2013; Michael, Berkman, Colditz, Holmes \& Kawachi, 2002; Paterson, Jones, Rattray \& Lauder, 2013). Tales resultados concuerdan con el postulado del apoyo social como un constructo que interactúa con la calidad de vida relacionada con la salud. Así, un mejor o mayor apoyo social tiene efectos directos y amortiguadores en el bienestar y el ajuste en el cáncer (Nausheen et al., 2009), lo que llevará a presentar una mejor calidad de vida.

Al compararse los pacientes con diagnóstico de cáncer de próstata con las pacientes de cáncer de mama, tener cáncer de mama reduce el puntaje total de la escala MOS, lo cual explica el pobre apoyo social que ellas tienen al momento del diagnóstico, resultado que no se puede comparar debido a la limitación de la literatura encontrada acerca del tema mencionado. Por ello se insta a realizar estudios en los cuales se estudie la asociación o capacidad de predicción que pueda tener el hecho de padecer un tipo de cáncer específico (próstata, mama, cuello uterino, gástrico, colon-recto, entre otros) y el apoyo social percibido.

La condición gregaria de los seres humanos nos impulsa a buscar a otros, a fin de dar y recibir apoyo. La empatía que sentimos hacia nuestros semejantes nos moviliza a ayudarlos en caso de necesidad, por ejemplo, en caso de crisis, urgencias o enfermedad (Nausheen et al., 2009; Thompson et al., 2013). De acuerdo con esto, se podría pensar que personas con diversas patologías obtengan mayor apoyo social o, como se observó, que la presencia de comorbilidades aumente el puntaje de la escala MOS; sin embargo, esta información debe ser manejada con cautela, pues hace falta mayor profundidad de análisis y variedad de estudios que aborden esta hipótesis.

Aunque la relación entre el apoyo social percibido y las variables sociodemográficas no resultó significativa, el apoyo social percibido es una variable importante y que interactúa de manera positiva con el constructo de calidad de vida en pacientes oncológicos; además, en momentos de crisis como enfermedad el apoyo social es un aspecto que cumple un papel relevante para las personas.

\section{Limitaciones}

Las principales limitaciones del estudio fueron el escaso tamaño de la muestra (sobre todo, en el número de pacientes por tipo de cáncer) y sus características (dado que la población que acude al Instituto Nacional de Cancerología suele ser de bajos recursos económicos y tener bajo nivel aca- 
démico). El tipo de estudio transversal limitó la realización de mediciones comparativas del apoyo social y la calidad de vida en distintos momentos, antes y después del tratamiento quirúrgico.

\section{Estudios futuros}

Los resultados encontrados en el presente estudio recalcan la importancia de continuar en la búsqueda de otros factores relacionados con el aumento del apoyo social y de la calidad de vida en pacientes con diagnóstico oncológico.

\section{Referencias}

Alonso, A., Menéndez, V., \& Graça, M. (2007). Adaptação cultural e validação da versão portuguesa Questionário Medical Outcomes Study Social Support Survey (MOS-SSS). Acta Médica Portuguesa, 20, 525-533.

Arias, S., Murillo, R., Piñeros, M., Bravo, M., Hernández, G., Pardo, C., ... Wiesner, C. (2008). Prioridades de investigación para el control del cáncer en Colombia. Revista Colombiana de Cancerología, 11(3), 152-164.

Cheng, H., Sit, J., Chan, C., So, W., Choi, K., \& Cheng, K. (2013). Social support and quality of life among Chinese breast cancer survivors: Findings from a mixed methods study. European Journal of Oncology Nursing, 17, 788-796.

Costa, G., Ballesteros, M., \& Gil, F. (2014). The influence of coping response and health-related quality of life on perceived social support during cancer treatment. Palliative and Supportive Care, (1-7).

Costa, G., Salamero, M., \& Gil, F. (2007). Validación del cuestionario MOS-SSS de apoyo social en pacientes con cáncer. Medicina Clínica (Barcelona), 128(18), 687-691.

Dapueto, J., Servente, L., Francolino, C., \& Hahn, E. (2005). Determinants of quality of life in patients with cancer. Cancer, 103(5), 1072-1081.
Evon, D., Esserman, D., Ramcharran, D., Bonner, J., \& Fried, M. (2011). Social support and clinical outcomes during antiviral therapy for chronic hepatitis C. Journal of Psychosomatic Research, 71, 349-356.

Fagundes, C., Alfano, C., Bennett, J., Glaser, R., Povoski, S., Lipari, A.,... Kiecolt-Glaser, J. (2012). Social Support and socioeconomic status interact to predict Epstein-Barr virus latency in women awaiting diagnosis or newly diagnosed with breast cancer. American Psychological Association, Health Psychology, 31(1), 11-19.

Gottlieb, B. \& Bergen, A. (2010). Social support concepts and measures. Journal of Psychosomatic Research, 69, 511-520.

Heinonen, H., Volin, L., Uutela, A., Zevon, M., Barrick, C., \& Ruutu, T. (2001). Gender-associated differences in the quality of life after allogeneic BMT. Bone Marrow Transplantation, 28, 503-509.

Huang, C., \& Hsu, M. (2013). Social support as a moderator between depressive symptoms and quality of life outcomes of breast cancer survivors. European Journal of Oncology Nursing, 17, 767-774.

Londoño, N., Rogers, H., Castilla, J., Posada, S., Ochoa, $N$. , Jaramillo, M., ... Aguirre, J. (2012). Validación en Colombia del cuestionario MOS de apoyo social. International Journal of Psychological Research, 5(1), 142-150.

Michael, Y., Berkman, L., Colditz, G., Holmes, M., $\&$ Kawachi, I. (2002). Social networks and health-related quality of life in breast cancer survivors: A prospective study. Journal of Psychosomatic Research, 52, 285-293.

Mickelson, K., \& Kubzansky, L. (2003). Social distribution of social support: The mediating role of life events. American Journal of Community Psychology, 32, 265-281.

Nausheen, B., Gidron, Y., Peveler, R., \& Moss-Morris, R. (2009). Social support and cancer 
progression: A systematic review. Journal of Psychosomatic Research, 67, 403-415.

Paterson, C., Jones, M., Rattray, J., \& Lauder, W.. (2013). Exploring the relationship between coping, social support and health-related quality of life for prostate cancer survivors: A review of the literature. European Journal of Oncology Nursing, 17, 750-759.

Rodríguez, J., Pastor, M., \& López, S. (1993). Afrontamiento, apoyo social, calidad de vida y enfermedad: Departamento de Psicología de la Salud. Psicothema, 5, 349-372.

Rodríguez, S., Rodríguez, E., \& Camelo, H. (2007). Validación argentina del cuestionario MOS de apoyo social percibido. Psicodebate, 7, 155-168.

Sánchez, R., Ballesteros, M., \& Arnold, B. J. (2011). Validation of the FACT-G scale for evaluating quality of life in cancer patients in Colombia. Quality of Life Research: An International Journal of Quality of Life Aspects of Treatment, Care and Rehabilitation, 20(1), 19-29. doi:doi. org/10.1007/s11136-010-9727-0

\section{Fecha de recepción: 15 de julio de 2014} Fecha de aceptación: 15 de abril de 2015
Sherbourne, C., \& Stewart, A. (1991). The MOS Social Support Survey. Social Science \& Medicine, 32(6), 705-714.

Terol M., C. (1999). Apoyo social y salud en pacientes oncológicos. Tesis doctoral, Universidad Miguel Hernández.

Thompson, T., Rodebaugh, T., Pérez, M., Schootman, M., \& Jeffe. D. B. (2013). Perceived social support change in patients with early stage breast cancer and controls. American Psychological Association, Health Psychology, 32(8), 886-895.

Waters, E., Liu, Y., Schootman, M., \& Jeffe, D. (2013). Worry about cancer progression and low perceived social support: Implications for quality of life among early-stage breast cancer patients. Annals of Behavioral Medicine. Author Manuscript, 45(1), 57-68.

World Health Organization. (1997). WHOQOL: Measuring quality of life. Geneva: World Health Organization. 